\title{
RESEARCH
}

Open Access

\section{Lectin complement pathway initiators after subarachnoid hemorrhage - an observational study}

Jeppe Sillesen Matzen ${ }^{1 *}$ (D), Charlotte Loumann Krogh ${ }^{1}$, Julie Lyng Forman², Peter Garred ${ }^{3,4}$, Kirsten Møller ${ }^{1,4}$ and Søren Bache ${ }^{1}$

\begin{abstract}
Background: This exploratory study investigated the time-course of lectin complement pathway (LCP) initiators in cerebrospinal fluid (CSF) and plasma in patients with subarachnoid hemorrhage (SAH), as well as their relationship to delayed cerebral ischemia (DCI) and functional outcome.

Methods: Concentrations of ficolin-1, ficolin-2, ficolin-3, and mannose-binding lectin (MBL) were analyzed in CSF and plasma from patients with SAH. Samples were collected daily from admission until day 9 (CSF; N_PATIENTS $=63$, $n_{\text {n_SAMPLES }}=399$ ) and day 8 (plasma; N_PATIENTS $=50$, n_SAMPLES $=358$ ), respectively. Twelve neurologically healthy patients undergoing spinal anesthesia and 12 healthy blood donors served as controls. The development of DCl during hospitalization and functional outcome at 3 months (modified Rankin Scale) were registered for patients.

Results: On admission, CSF levels of all LCP initiators were increased in SAH patients compared with healthy controls. Levels declined gradually over days in patients; however, a biphasic course was observed for ficolin-1. Increased CSF levels of all LCP initiators were associated with a poor functional outcome in univariate analyses. This relationship persisted for ficolin-1 and MBL in multivariate analysis after adjustments for confounders (age, sex, clinical severity, distribution and amount of blood on CT-imaging) and multiple testing $(1.87 \mathrm{ng} / \mathrm{mL}$ higher in average, $95 \% \mathrm{Cl}, 1.17$ to 2.99 and $1.69 \mathrm{ng} / \mathrm{mL}$ higher in average, $95 \% \mathrm{Cl}, 1.09$ to 2.63, respectively). In patients who developed DCl compared with those without DCI, CSF levels of ficolin-1 and MBL tended to increase slightly more over time (p_interaction $=0.021$ and 0.033 , respectively); however, no association was found after adjustments for confounders and multiple testing ( $p$-adj_interaction $=0.086$ and 0.098, respectively). Plasma ficolin- 1 and ficolin-3 were lower in SAH patients compared with healthy controls on all days. $\mathrm{DCl}$ and functional outcome were not associated with LCP initiator levels in plasma.
\end{abstract}

Conclusion: Patients with SAH displayed elevated CSF levels of ficolin-1, ficolin-2, ficolin-3, and MBL. Increased CSF levels of ficolin-1 and MBL were associated with a poor functional outcome.

(Continued on next page)

\footnotetext{
* Correspondence: jeppe.sillesen.matzen@gmail.com

'Department of Neuroanaesthesiology, The Neuroscience Centre,

Rigshospitalet, University of Copenhagen, Blegdamsvej 3, 2100 Copenhagen

$\varnothing$, Denmark

Full list of author information is available at the end of the article
}

(C) The Author(s). 2020 Open Access This article is licensed under a Creative Commons Attribution 4.0 International License, which permits use, sharing, adaptation, distribution and reproduction in any medium or format, as long as you give appropriate credit to the original author(s) and the source, provide a link to the Creative Commons licence, and indicate if changes were made. The images or other third party material in this article are included in the article's Creative Commons licence, unless indicated otherwise in a credit line to the material. If material is not included in the article's Creative Commons licence and your intended use is not permitted by statutory regulation or exceeds the permitted use, you will need to obtain permission directly from the copyright holder. To view a copy of this licence, visit http://creativecommons.org/licenses/by/4.0/ The Creative Commons Public Domain Dedication waiver (http://creativecommons.org/publicdomain/zero/1.0/) applies to the data made available in this article, unless otherwise stated in a credit line to the data. 
(Continued from previous page)

Trial registration: This study was a retrospective analysis of samples, which had been prospectively sampled and stored in a biobank. Registered at clinicaltrials.gov (NCT01791257, February 13, 2013, and NCT02320539, December 19, 2014).

Keywords: Ficolin, Lectin complement pathway, Subarachnoid hemorrhage, Delayed cerebral ischemia, Functional outcome

\section{Background}

Aneurismal subarachnoid hemorrhage (SAH) is a lifethreatening condition accounting for $\sim 5 \%$ of all stroke cases. Despite low annual incidence rates [1], the total loss of productive life years in the SAH patients is similar to more common types of stroke [2], due to a high mortality rate [1] and a low chance that survivors return to the labor market $[2,3]$.

Delayed cerebral ischemia (DCI) is a main contributor to a poor functional outcome in patients surviving $\mathrm{SAH}$ $[4,5]$. The pathogenesis and pathophysiology of DCI are still incompletely understood. Previously, DCI has been attributed to cerebral vasospasm [6], but recent research suggests a multifactorial origin, including neuroinflammation as a central causal factor [7, 8].

The complement system is part of the innate immune system, which comprises a first-line defense against invading microorganisms, enabling clearance of dying cells, and amplifying adaptive and inflammatory immune responses upon stimulation [9]. Three pathways activate the complement system, i.e., the classical, the alternative, and the lectin complement pathway (LCP) [10]. The initiators of the LCP, comprising ficolin-1, ficolin-2, and ficolin-3, mannose-binding lectin (MBL), and collectin10 and collectin-11, induce down-streams activation upon binding to specific carbohydrate structures on injured cells and microorganisms [11, 12].

Recent studies have reported an association between LCP initiators in plasma and functional outcome in ischemic stroke patients [13-16]. LCP initiators have previously been investigated in SAH patients but with conflicting results [17-20].

We aimed to investigate the time-course of LCP initiator levels in CSF and plasma after SAH and to describe their potential relationship to DCI and functional outcome.

\section{Methods}

\section{Standard protocol approvals, registrations, and patient consents}

This study was a retrospective analysis of samples from CSF and plasma, which had been prospectively sampled and stored in a biobank, and some of which were included in a prospective observational cohort study of microRNAs [21]. Protocols were approved by the Danish Scientific Ethics Committee of the Capital Region (H-3-2013-009 and H-3-
2014-073) including amendments for the present study (H3-2013-009 and H-6-2014-073) and the Danish Data Protection Agency (I-suite \# 02116 and 03279) and registered at clinicaltrials.gov (NCT01791257 and NCT02320539). Informed consent was obtained from the next of kin, the general practitioner and, when possible, from the patient.

This manuscript adheres to requirements set by the STrengthening the Reporting of OBservational studies in Epidemiology (STROBE) statement.

\section{Study participants}

Patients with spontaneous aneurysmal subarachnoid hemorrhage (SAH) as diagnosed by computed tomography (CT) and CT angiography who were admitted to the Neurointensive Care Unit at Rigshospitalet (Copenhagen, Denmark) were assessed for eligibility. Inclusion criteria were $>18$ years of age. Exclusion criteria were more than $24 \mathrm{~h}$ uncertainty of the time of ictus, as well as the inability to speak and understand Danish.

Neurologically healthy patients $>18$ years of age with an American Society of Anesthesiologists physical status classification $\leq 2$, who had spinal anesthesia before lower limb orthopedic surgery at Frederiksberg Hospital, were included and served as the control group for the CSF analyses.

Neurologically healthy blood donors $>18$ years of age were included from the National Donor Registry (Copenhagen, Denmark) and served as the control group for the plasma analyses.

\section{Clinical definitions and outcome measures}

The clinical severity of SAH was assessed using the World Federation of Neurosurgical Societies (WFNS) grading scale, which categorizes patients from 1 to 5 ; 1 indicating the lowest and 5 indicating the highest disease severity [22]. The extent of intracranial hemorrhage on admission $\mathrm{CT}$ was registered by the Fisher grading scale, which rates the severity of SAH from 1 to 4 based on the distribution and amount of blood on CT scans of the head [23].

Clinical deterioration due to DCI was defined according to Vergouwen et al. as the development of a new neurological deficit or a decrease of at least 2 points in GCS, persisting for at least $1 \mathrm{~h}$, and which was not explained by any other potential cause [5]. Two clinicians independently reviewed all patient data obtained in the medical journal within 3 weeks from ictus and allocated 
the patients to five subgroups regarding the diagnostic certainty of DCI (definite DCI, probably DCI, possible $\mathrm{DCI}$, probably not $\mathrm{DCI}$, and definitely not $\mathrm{DCI})$. To increase the chance of detecting a difference between groups, only patients from the groups, 'definite DCI' and 'definitely not DCI' were compared.

Functional outcome at 3-months follow-up was based on the modified ranking scale (mRS) [24], which measures the degree of disability (i.e., dependence in daily activities) after a stroke ranging from a mRS score of 0 (no disability symptoms) to 6 (death). This outcome was subsequently dichotomized into good functional outcome, defined as independence or minor disability ( $\mathrm{mRS}$ $0-2$ ), and poor functional outcome, defined as being dependent or dead (mRS 3-6).

\section{CSF samples}

From patients with an external ventricular drain (EVD), CSF was collected immediately after placement with a sterile procedure, and once daily using an aseptic procedure the following 7 days. From neurologically healthy patients, one CSF sample was collected using a sterile procedure immediately before spinal anesthesia for orthopedic lower limb surgery. CSF samples were immediately put on ice and spun within $15 \mathrm{~min}$ at $500 \mathrm{~g}$ for $10 \mathrm{~min}$, and the supernatant was stored at $-80^{\circ} \mathrm{C}$.

\section{Plasma samples}

Blood was collected from an arterial catheter from SAH patients upon admission and once daily the following 7 days. If no arterial catheter was present, sampling was done from a central venous catheter. From healthy volunteers, blood was sampled once during a blood donation. Blood samples were immediately put on ice and spun within $15 \mathrm{~min}$ at $2000 \mathrm{~g}$ for $10 \mathrm{~min}$, and the plasma samples were stored at $-80^{\circ} \mathrm{C}$. Both plasma and CSF samples were collected at the same time of the morning each day.

\section{Protein quantification}

The concentration of LCP initiators (ficolin-1, ficolin-2, ficolin-3, and MBL) in CSF and plasma was determined using sandwich enzyme-linked immunosorbent assay (ELISA) developed in the Laboratory of Molecular Medicine, Rigshospitalet, according to previously described procedures [25-28]. Briefly, microtiter plates (Nunc Immuno Plates, F384 Maxisorp) were coated with one of the following monoclonal antibodies against ficolin-1 (HP9039; Hycult Biotech, Uden, Nederlands) and inhouse produced biotinylated antibodies against ficolin-2 (anti-ficolin 2 ficolin-219), ficolin-3 (anti-ficolin 3 ficolin-334), and MBL (anti-MBL Hyb-131-1) and diluted in phosphate-buffered saline $+0.05 \%$ Tween-20 (PBS-T), and incubated overnight. Samples were diluted 1:200 and run in triplicates and parallel to a standard pool with a known concentration. Subsequently, polyclonal donkey anti-rapid antibody (ficolin-1) or streptavidinhorseradish peroxidase conjugate (ficolin-2, ficolin-3, and MBL) was applied and plates were developed using ophenylenediamine dihydrochloride substrate and stopped by adding $\mathrm{H}_{2} \mathrm{SO}_{4}$. Assays were optimized for automated analysis in a 384-well format using the Biomek FX platform (Beckman Coulter, Fullerton, California, USA) for pipetting followed by a DTX880 Multimode detector system where assays were analyzed at $490 \mathrm{~nm}$.

The lower detection limit was as follows in plasma: 5 $\mathrm{ng} / \mathrm{ml}$ for ficolin-1, $0.005 \mu \mathrm{g} / \mathrm{ml}(5 \mathrm{ng} / \mathrm{ml})$ for ficolin-2, $1 \mathrm{ng} / \mathrm{ml}$ for ficolin-3, and $20 \mathrm{ng} / \mathrm{ml}$ for MBL; it was 0.4 $\mathrm{ng} / \mathrm{ml}$ in CSF for all four biomarkers. We obtained the following coefficients of variation as calculated from the raw triplicate measurements: Ficolin-1: CSF 9.3\%, plasma 7.1\%; ficolin-2: CSF 20.2\%, plasma 10.6\%; ficolin-3: CSF 18.1\%, plasma 9.6\%, MBL: CSF 9.2\%, plasma $7.7 \%$.

\section{Statistical analysis}

Plasma and CSF levels of LCP initiators on admission and specific days after the initial bleeding in SAH patients were compared with those of neurologically healthy individuals using Wilcoxon's rank-sum test. Within SAH patients, levels were compared between the groups, 'definitely $\mathrm{DCl}$ vs 'definitely not DCI' and 'poor functional outcome' vs 'good functional outcome', using a linear mixed model with subgroup (DCI or functional outcome) and day as fixed effects, and patients as a random effect. Crude comparison was done without including any further covariates (univariate analyses), whereas adjusted analyses included sex, age, and WFNS and Fisher scores as covariates (multivariate analyses). The linear mixed model was applied twice: (1) Assuming no interaction (i.e., assuming that LCP initiator levels did not develop differently over time between groups) thereby evaluating the overall difference in concentrations (p), and (2) including the group*day interaction to explore potentially different time developments between groups ( $\mathrm{p}_{-}$ interaction). Goodness of fit was assessed by residual plots. Due to substantial skewness, CSF levels were logtransformed prior to analysis and results were expressed accordingly (i.e., as percentwise differences in medians). $P$ values were corrected for multiple testing using the Benjamini-Hochberg procedure.

All statistical analysis was performed using Statistical Analysis System v.9.4 (SAS Institute Inc., Cary, NC).

\section{Results}

In total, 82 patients were recruited for this study and included in the CSF study alone $(N=32)$, the plasma study alone $(N=19)$, or both $(N=31)$ (Table 1$)$. The $63 \mathrm{SAH}$ patients in the CSF study all underwent placement of an EVD for clinical reasons and had a total of 402 CSF 
Table 1 Demographic data of the SAH patients and related control groups

\begin{tabular}{|c|c|c|c|c|}
\hline & $\begin{array}{l}\text { CSF control group } \\
(n=12)\end{array}$ & $\begin{array}{l}\text { CSF } \\
(n=63)\end{array}$ & $\begin{array}{l}\text { Plasma Control Group } \\
(n=12)\end{array}$ & $\begin{array}{l}\text { Plasma } \\
(n=50)\end{array}$ \\
\hline Age, mean years (SD) & $69( \pm 9)$ & $59( \pm 11)$ & $55( \pm 7)$ & $61( \pm 11)$ \\
\hline \multicolumn{5}{|l|}{ Sex, $n(\%)$} \\
\hline Men & $8(66.7)$ & $14(22.2)$ & $4(33.3)$ & $5(10)$ \\
\hline Women & $4(33.3)$ & $49(77.8)$ & $8(66,7)$ & $45(90)$ \\
\hline \multicolumn{5}{|l|}{ WFNS, $n(\%)$} \\
\hline 1 & & $8(12.7)$ & & $17(34.0)$ \\
\hline 2 & & $12(19.0)$ & & $8(16.0)$ \\
\hline 3 & & $4(6.3)$ & & $5(10.0)$ \\
\hline 4 & & $21(33.3)$ & & $10(20.0)$ \\
\hline 5 & & 18 (28.6) & & $10(20.0)$ \\
\hline \multicolumn{5}{|l|}{ Fisher score, $n(\%)$} \\
\hline 1 & & $0(0)$ & & $0(0)$ \\
\hline 2 & & $1(1.6)$ & & $4(8.0)$ \\
\hline 3 & & $48(77.4)$ & & $37(74.0)$ \\
\hline 4 & & $13(21.0)$ & & $9(18.0)$ \\
\hline Definite $\mathrm{DCl}, n(\%)$ & & $18(28.6)$ & & $13(26.0)$ \\
\hline Definitely not $\mathrm{DCl}, n(\%)$ & & $18(28.6)$ & & $20(40.0)$ \\
\hline Days from ictus to $\mathrm{DCl}$, mean (SD) & & $6.5( \pm 1.5)$ & & $7.2( \pm 2.2)$ \\
\hline \multicolumn{5}{|l|}{ mRS, $n(\%)$} \\
\hline $0-2$ & & $34(54.0)$ & & $35(70.0)$ \\
\hline 3-6 (poor functional outcome) & & $29(46.0)$ & & $15(30.0)$ \\
\hline \multicolumn{5}{|l|}{ Aneurysm location, $n(\%)$} \\
\hline Anterior cerebral artery & & $25(39.7)$ & & $16(32.0)$ \\
\hline Middle cerebral artery & & $13(20.6)$ & & $10(20.0)$ \\
\hline Posterior cerebral artery & & $16(25.4)$ & & $18(36.0$ \\
\hline Internal carotid artery & & $9(14.3)$ & & $6(12.0)$ \\
\hline \multicolumn{5}{|c|}{ Technique for securing aneurysm, $n(\%)$} \\
\hline Coil & & $42(66.7)$ & & $35(71.4$ \\
\hline Clip & & $21(33.3)$ & & $14(28.6)$ \\
\hline \multicolumn{5}{|l|}{ Vasospasm, $n(\%)$} \\
\hline Yes & & $28(73.7)$ & & $17(70.8)$ \\
\hline No & & $10(26.3)$ & & $7(29.2)$ \\
\hline
\end{tabular}

CSF cerebrospinal fluid, WFNS World Federation of Neurological Surgeons scale, EBI early brain injury, DCl delayed cerebral ischemia, $m R S$ Modified ranking scale

samples collected (Sup. Fig. 1), of which 399 were used for final analysis. Of these 63 patients, 39 (62\%) were in a poor condition on admission (WFNS grade 4 or 5 ) and 13 (21\%) had a Fisher grade of 4 (i.e., intraventricular hemorrhage or intracerebral hemorrhage). Thirty-six were assessable for development of DCI; of these, 18 were categorized as 'definite' and 18 as 'definitely not DCI'. Clinical deterioration due to DCI typically occurred between day 6 and day 7 (mean $=6.5$ days, $\mathrm{SD}=1.5)$. At the 3 -month follow-up, all $63 \mathrm{SAH}$ patients were assessable for a functional outcome, of whom 29 (46\%) had a poor (mRS 3-6) and $34(54 \%)$ had a good functional outcome (mRS 0-2), respectively. Twelve CSF samples were collected from 12 neurologically healthy patients.

From the 50 patients in the plasma study, 359 plasma samples were collected (Sup. Fig. 2), of which 358 were used for final analysis. Of these $50 \mathrm{SAH}$ patients, 20 (40\%) were in a clinically poor condition (WFNS 4-5) on admission and 9 (18\%) had a Fisher grade of 4 (i.e., intraventricular hemorrhage or intracerebral hemorrhage). Thirty-three SAH patients were assessable for the development of DCI, of which 13 patients were categorized as 'definite' and 20 patients as 'definitely not' DCI. On average, clinical deterioration due to DCI occurred on 
day 7 (mean $=7.2, \mathrm{SD}=2.2$ ). At the 3-month follow-up, all $50 \mathrm{SAH}$ patients were assessable for the functional outcome, of whom 15 (30\%) had a poor and $35(70 \%)$ had a good functional outcome, respectively. Twelve plasma samples were collected from 12 healthy volunteers.

\section{LCP initiator concentrations in SAH patients vs healthy controls (Fig. 1)}

Ficolin-1, ficolin-2, and MBL were not detected in CSF from neurologically healthy patients and ficolin-3 concentrations in CSF were low compared with $\mathrm{SAH}$ patients. Median levels of ficolin-1, ficolin-2, and ficolin-3, and MBL were elevated in CSF on all days after SAH compared with neurologically healthy patients; concentrations were high on admission followed by a gradual decline towards normal levels on day 7 to 9 . In contrast, ficolin-1 showed a biphasic slope with a second peak between day 3 and 9.

Average plasma concentrations of all LCP initiators in SAH patients were lower than or comparable to the levels of neurologically healthy individuals. Ficolin-1 and ficolin-3 were lower on all days after SAH, whereas ficolin-2 and MBL were lower only on days 1-2 and 14 , respectively. Ficolin-2 showed a biphasic slope with a second peak from day 2 . Ficolin-1 showed a minor decline between day 0 and day 2 followed by a gradual day-by-day increase. MBL showed a gradual increase over time, reaching normal levels from day 5 . Ficolin-3 remained low on all days.

Daily levels of the LCP initiators in SAH and neurologically healthy patients are shown in Fig. 1.

\section{DCI - longitudinal analysis (Fig. 2 and Table 2)}

For the 36 patients in the CSF study who were diagnosed as either 'definitely DCI' or 'definitely not DCI', 241 CSF samples were available.

In univariate analysis, the CSF level of ficolin-1 seemed elevated in patients with DCI versus patients without DCI $(1.87 \mathrm{ng} / \mathrm{mL}$ higher in average, 95\% confidence interval [CI], 0.99 to $3.53, p=0.052$ ), although insignificant. However, after adjustment for multiple testing, the association was no longer found ( $\mathrm{p}$-adj $=0.20$ ). In multivariate analysis adjusted for sex, age, WFNS, and Fisher score no association was found.

Ficolin-1 and MBL increased slightly more over time in patients with DCI versus without DCI, i.e., a different slope was present (p_interaction $=0.021$ and 0.033, respectively; data not shown). Adjusted for confounders (sex, age, WFNS, and Fisher score), the increase over time for ficolin-1 and MBL remained significant, but with further adjustment for multiple testing, the increase over time became non-significant ( $\mathrm{p}$-adj_interaction $=$ 0.086 and 0.098 , respectively, Fig. 2).
Ficolin-2 and ficolin-3 in CSF did not differ between patients with and without DCI.

For the 33 patients who were diagnosed with either 'definitely DCI' or 'definitely not DCI', 233 plasma samples were available. Neither overall difference in plasma concentrations (Sup. Table 1) nor overall changes over time was observed for any of the LCP initiators (ficolin1 , ficolin-2, ficolin-3, and MBL) in patients with or without DCI (Fig. 2).

\section{Functional outcome - longitudinal analysis (Fig. 3 and Table 2)}

All 399 CSF samples and all 358 plasma samples were entered into these analyses.

In univariate analyses, CSF levels of ficolin-1, ficolin-2, ficolin-3, and MBL were higher in patients with a poor functional outcome compared with those with a good functional outcome. In multivariate analyses adjusting for sex, age, WFNS, and Fisher score as well as for multiple testing, ficolin-1 and MBL levels remained higher in patients with a poor functional outcome $(1.87 \mathrm{ng} / \mathrm{mL}$ higher in average, $95 \% \mathrm{CI}, 1.17$ to $2.99, p=0.040)$ and $1.69 \mathrm{ng} /$ $\mathrm{mL}$ higher in average, $95 \% \mathrm{CI}, 1.09$ to $2.63, p=0.040$ ).

No change over time was detected between groups, indicating that the slopes were parallel (p_interaction $>0.05$, data not shown).

In contrast, LCP initiators in plasma did not differ between patients with good and those with poor functional outcomes either overall or by time (Fig. 3 and Sup. Table 1).

\section{Discussion}

We investigated the day-by-day changes of LCP initiators in both CSF and plasma after SAH. Our study revealed three major findings. First, CSF concentrations of LCP initiators were increased throughout the first week following $\mathrm{SAH}$, followed by a gradual decline over time. Second, increased CSF levels of all investigated LCP initiators, especially ficolin-1 and MBL, were associated with a poor functional outcome in SAH patients. Third, in univariate analysis, patients with DCI compared with those without DCI, no association was found between DCI and overall LCP initiator levels in CSF. However, ficolin-1 tended to increase slightly more over time in patients with DCI than without (i.e., slopes showing CSF levels increased more over time in the DCI group versus the group without DCI), but the increase was not significant after adjustment for multiple testing (p-adj_interaction $=0.086$, Fig. 2) .

The present design does not allow us to conclude whether the increased CSF concentration of LCP initiators in the present study is due to local synthesis or simply to a spill-over from the systemic circulation, e.g., due to bloodbrain barrier (BBB) dysfunction after SAH. In favor of spill-over from plasma, invasion of complement and 


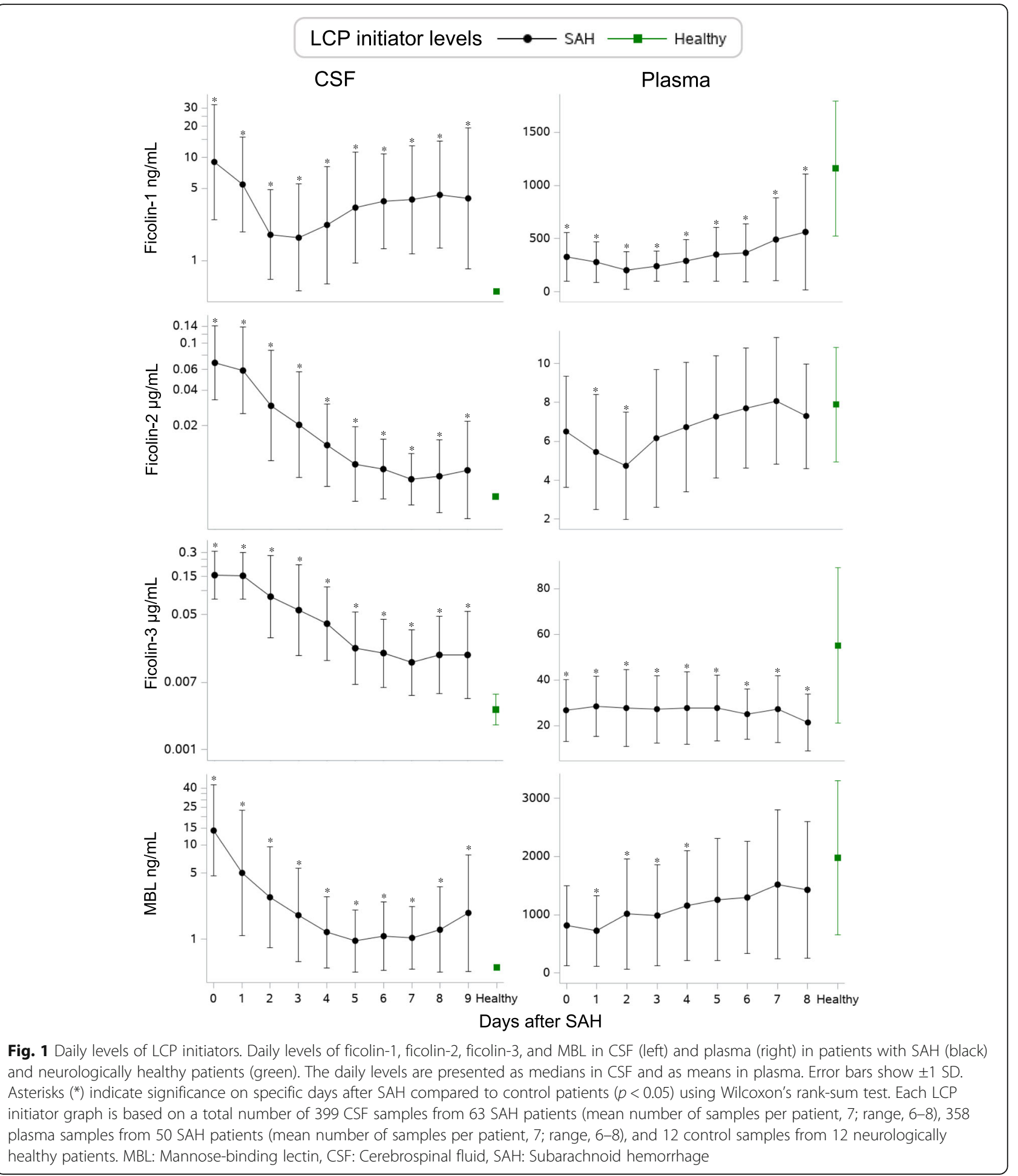

inflammatory cells over the disrupted $\mathrm{BBB}$ has been proposed as a major contributor to the BBB pathology in other neuroinflammatory central nervous system (CNS) disorders, such as traumatic brain injury, bacterial meningitis, and cerebral malaria [29]. However, this suggestion was based on experimental findings rather than clinical data.
Our reported median CSF level of MBL of $15 \mathrm{ng} / \mathrm{ml}$ is slightly lower than the median level of approximately 25 $\mathrm{ng} / \mathrm{ml}$ in patients with pneumococcal meningitis and no MBL deficiency reported in a study evaluating CSF levels of MBL in relation to pneumococcal meningitis, but slightly higher than in such patients with MBL deficiency 


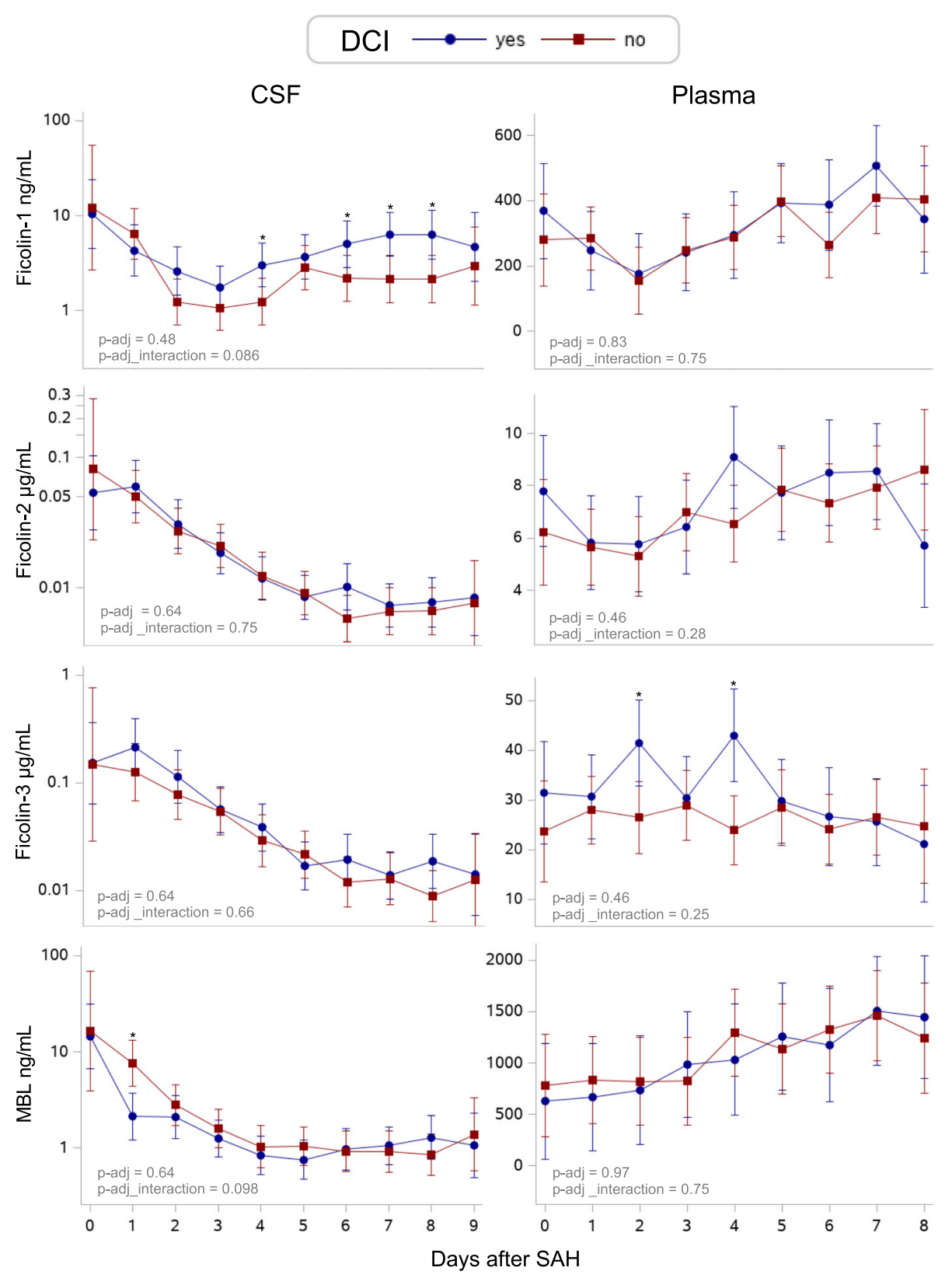

Fig. 2 Predicted daily LCP initiator levels in SAH patients with or without the development of DCI. Predicted daily levels of ficolin-1, ficolin-2, ficolin-3, and MBL in CSF (left) and in plasma (right) in patients with (blue) or without (red) the development of DCI. The predicted daily levels are presented as medians in CSF and as means in plasma. Error bars show 95\% confidence intervals. $P$ values shown in the bottom left of each graph are adjusted for confounders (age, sex, WFNS, and Fisher score) and multiple testing (Benjamini-Hochberg procedure) and represent the overall difference in concentration between groups ( $p$-adj) and overall different development over time ( $p$-adj_interaction) for each LCP initiator. Asterisks indicate $\left(^{*}\right)$ significant levels on specific days $<0.05$. Each LCP initiator graph is based on a total number of 241 CSF samples from 36 SAH patients (mean number of samples per patient, 7; range, 6-8) and 233 plasma samples from 33 SAH patients (mean number of samples per patient, 7; range, 6-8). LCP: Lectin complement pathway, MBL: Mannose-binding lectin, CSF: Cerebrospinal fluid, CT: Computed tomography, DCl: Delayed cerebral ischemia, SAH: Subarachnoid hemorrhage

( $<5-15 \mathrm{ng} / \mathrm{ml}$ depending on genotype) [30]. Furthermore, another study reported a mean CSF MBL level of $5.11 \mathrm{ng} /$ $\mathrm{ml}$ in patients with non-HIV-associated cryptococcal meningitis [31]. To our knowledge, nobody has reported CSF levels of ficolins in pneumococcal meningitis, nor CSF levels of MBL or ficolins in patients with cerebral malaria or traumatic brain injury. In favor of local production of complement proteins, production of initiators and downstream mediators of the classical and the alternative pathway does occur in the healthy brain $[32,33]$. Most LCP 
Table 2 Overall difference in CSF levels of each LCP initiator in SAH subgroups (DCI and functional outcome)

\begin{tabular}{|c|c|c|c|c|c|c|}
\hline \multirow[b]{2}{*}{ CSF-levels } & \multicolumn{3}{|c|}{ Univariate analyses } & \multicolumn{3}{|c|}{$\begin{array}{l}\text { Multivariate analyses } \\
\text { (age, sex, WFNS, Fisher) }\end{array}$} \\
\hline & $\begin{array}{l}\text { Estimate } \\
(95 \% \mathrm{Cl})\end{array}$ & $\mathrm{p}$ & p-adj & $\begin{array}{l}\text { Estimate } \\
(95 \% \mathrm{Cl})\end{array}$ & $\mathbf{p}$ & p-adj \\
\hline \multicolumn{7}{|l|}{$\mathrm{DCl}$} \\
\hline $\begin{array}{l}\text { Ficolin-1 } \\
(\mathrm{ng} / \mathrm{mL})\end{array}$ & $1.87(0.99,3.53)$ & 0.052 & 0.20 & $1.72(0.85,3.48)$ & 0.12 & 0.48 \\
\hline $\begin{array}{l}\text { Ficolin-2 } \\
(\mu \mathrm{g} / \mathrm{mL})\end{array}$ & $1.08(0.73,1.60)$ & 0.69 & 0.69 & $1.11(0.70,1.78)$ & 0.64 & 0.64 \\
\hline $\begin{array}{l}\text { Ficolin-3 } \\
(\mu \mathrm{g} / \mathrm{mL})\end{array}$ & $1.26(0.75,2.13)$ & 0.37 & 0.61 & $1.20(0.65,2.23)$ & 0.55 & 0.64 \\
\hline $\begin{array}{l}\mathrm{MBL} \\
(\mathrm{ng} / \mathrm{mL})\end{array}$ & $1.21(0.49,1.39)$ & 0.46 & 0.61 & $0.78(0.42,1.47)$ & 0.43 & 0.64 \\
\hline \multicolumn{7}{|c|}{ Functional outcome } \\
\hline $\begin{array}{l}\text { Ficolin-1 } \\
\text { (ng/mL) }\end{array}$ & $1.85(1.12,3.05)$ & $0.017^{*}$ & $0.022^{*}$ & $1.87(1.17,2.99)$ & $0.010^{*}$ & $0.040 *$ \\
\hline $\begin{array}{l}\text { Ficolin-2 } \\
(\mu \mathrm{g} / \mathrm{mL})\end{array}$ & $1.47(1.08,2.01)$ & $0.015^{*}$ & $0.022^{*}$ & $1.33(0.96,1,83)$ & 0.079 & 0.10 \\
\hline $\begin{array}{l}\text { Ficolin-3 } \\
(\mu \mathrm{g} / \mathrm{mL})\end{array}$ & $1.54(1.01,2.36)$ & $0.043^{*}$ & $0.043^{*}$ & $1.45(0.92,2.27)$ & 0.10 & 0.10 \\
\hline $\begin{array}{l}\mathrm{MBL} \\
(\mathrm{ng} / \mathrm{mL})\end{array}$ & $1.69(1.13,2.52)$ & $0.012^{*}$ & $0.022^{*}$ & $1.69(1.09,2.63)$ & $0.020^{*}$ & $0.040 *$ \\
\hline
\end{tabular}

Estimates represent the relative overall difference in CSF concentration (relative $\triangle$ medians) of each LCP initiator in patients with DCI versus without DCI (reference) and in patients with a poor versus good (reference) functional outcome, respectively. Both univariate linear mixed model analyses (left) and multivariate analyses (right) with adjustment for confounders are shown in the table.

$P$ values $(p)$ represent the overall difference in CSF concentrations between groups (assuming no interaction). $\mathrm{p}$-adj represent $p$ values corrected for multiple testing using Benjamini-Hochberg's procedure. Asterisks indicate significance levels $<0.05$.

$\Delta$ : Difference; $C$ confidence interval, CSF cerebrospinal fluid, Fisher: extent and location of the intracranial hemorrhage, $L C P$ lectin pathway initiators, MBL mannose-binding lectin; WFNS: clinical severity on admission

initiators (ficolin-2, ficolin-3, and MBL) are primarily produced by hepatocytes in the liver, after which they enter the systemic circulation, forming complexes with mannose-associated serine proteases (MASPs) [34]. Ficolin-1, in contrast, acts as a secretory protein and is mainly produced by the myeloid cell line. Ficolin- 1 is found in secretory granules in neutrophils and monocytes, where it can be exocytosed into extracellular environments upon stimulation [35]. We speculate that ficolin-1 is released by immune cells locally within the brain, whereas the remaining LCP initiators enter the intrathecal space from the blood. This hypothesis is supported by the finding that CSF ficolin-1 increased again after an initial decline. Although a concomitant increase in plasma levels could also have facilitated a larger passive influx, the plasma level of ficolin-2 increased in parallel with ficolin-1 but was not associated with an increase in CSF.

One other study has looked at LCP initiator levels in CSF after SAH [17]. The authors reported a tendency towards lower ficolin-1 levels in CSF after $24 \mathrm{~h}$ in $18 \mathrm{SAH}$ patients with angiographic vasospasm but did not find any association with the cerebral ischemic lesions. We were not able to directly compare the predicted concentrations in CSF in our study with the previous one because the outcome variables differed (cerebral ischemic lesions versus DCI). However, the previous study found a considerably higher median concentration of ficolin-1 in CSF from patients with cerebral ischemic lesions (51 ng/ $\mathrm{mL}$ (30-102)) compared with patients with DCI in our study $(4 \mathrm{ng} / \mathrm{mL}(2-8)$ on day 1$)$. Notably, this could be explained by a different distribution of patients with intraventricular hemorrhage; as many as $84 \%$ had intraventricular hemorrhage (i.e., original Fisher scale with grade 4) compared with $21 \%$ in the present study, indicating less contamination by blood in our CSF samples. Additionally, the studies used different protein quantification assays.

A study assessing the involvement of the LCP initiators in contused human brain tissue from patients with head trauma found that the LCP initiators were present inside and outside of brain vessels in all contusions examined [36]. Only ficolin-1 was found in normal brain tissue from control patients. In contrast, we were not able to detect ficolin-1 in CSF from neurologically healthy patients. One explanation to this may be that ficolin-1 acts as a secretory protein in the brain, which is only released into the extracellular compartment in response to inflammatory stimulation.

Another study examining brain samples in patients with acute ischemic stroke showed that certain complement proteins, including $\mathrm{MBL}$, were deposited in ischemic lesions in the brain [37]. The authors suggested that 


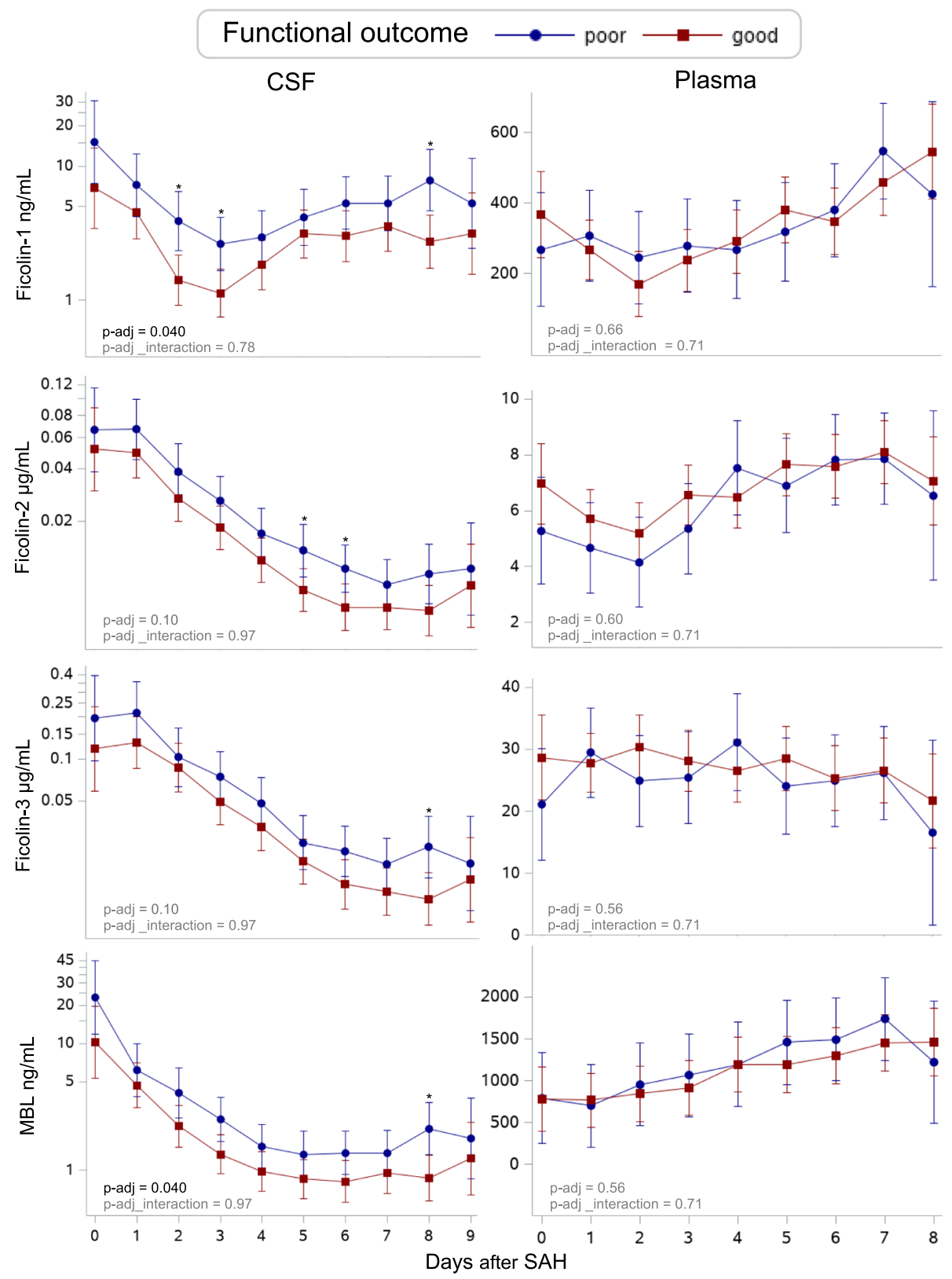

Fig. 3 Predicted daily levels of LCP initiators in SAH patients with poor versus good functional outcome. Predicted daily levels of ficolin-1, ficolin-2, ficolin-3, and MBL in CSF (left) and in plasma (right) in patients with a poor (blue) and good (red) functional outcome. The predicted daily levels are presented as medians in CSF and as means in plasma. Error bars show 95\% confidence intervals. $P$ values shown in the bottom left of each graph are adjusted for confounders (age, sex, WFNS, and Fisher score) and multiple testing (Benjamini-Hochberg procedure) and represent the overall difference in concentration between groups ( $p$-adj) and overall different development over time (p-adj_interaction) for each LCP initiator. Asterisks (*) indicate significant levels on specific days $<0.05$. Each LCP initiator graph is based on a total number of 399 CSF samples from 63 SAH patients (mean number of samples per patient, 7; range, 6-8) and 358 plasma samples from 50 SAH patients (mean number of samples per patient, 7; range, 6-8). MBL: Mannan-binding lectin, CSF: Cerebrospinal fluid, SAH = subarachnoid hemorrhage

increased complement deposition in the brain combined with decreased expression of complement regulators may be a possible mechanism of tissue damage during ischemia. Our findings of increased levels of LCP initiators in CSF associated with SAH and with poor functional outcome appear to support these findings. In line with this, experimental stroke models have reported that complement proteins mediate inflammation after cerebral ischemic injury and that inhibition of complement activation conferred neuronal protection and reduced stroke volume [38-41]. Our findings of increased levels of ficolin-1 and MBL associated with a poor functional outcome may support this 
notion; however, we have observed an association only and are unable to shed further light on these hypotheses.

Plasma levels of all investigated LCP initiators were lower or comparable in SAH patients compared with healthy individuals, and ficolin-1 and ficolin-3 were lower in both the hyperacute, acute, and the intermediate phase after SAH. Also, circulating LCP initiators were associated neither to DCI nor to functional outcome.

Few research groups have examined the changes of LCP initiators in plasma after $\mathrm{SAH}$, and conflicting results have been reported $[14,17,18,20]$. As a common denominator, the focus has primarily been on the hyperacute phase of SAH (i.e., hours after ictus). The studies used different approaches (e.g., regarding time from SAH to sampling, definition of DCI, techniques of protein quantification, etc.), which limits the comparability with our study.

This study is the first to provide information about the day-by-day changes of LCP initiators after SAH. The high number of consecutive samples of both CSF and plasma from each patient yielded a higher resolution than previous studies, conferring improved robustness and precision of the natural course of LCP initiators after SAH.

Furthermore, our study complies with the recent reporting recommendations in cellular and molecular SAH studies using recommended methods of sample acquisition, use of collection tubes, biospecimen processing and storage, inclusion of a neurologically healthy control group for both CSF and plasma, as well as using functional outcome and DCI as outcome measures [42]. This way, we hope to contribute to increased homogeneity across studies, which is essential for future biomarker validation to improve risk prediction and optimize treatment. Despite the retrospective study design, patients were thoroughly evaluated for the occurrence of DCI, as well as for their functional outcome 3 months after SAH.

However, this study also has noteworthy limitations. First, we evaluated multiple outcome measures (ficolin1, ficolin-2, ficolin-3, and MBL) which increase the risk of chance findings. We reduced this risk by correcting for multiple testing.

Due to the post hoc nature of this study, we cannot exclude a potential risk of selection bias as well as a risk of analytical variation due to the chosen method to detect LCP initiators. Furthermore, the retrospective design of the study rendered us unable to measure the actual activity of the LCP system and establish any potential causal relationship with the levels of LCP initiators. However, another study found plasma ficolin-3 and ficolin-3-mediated functional LCP activity to be well correlated [19]. Similarly, a causal relationship between LCP initiators and brain damage is not possible to determine in our study. Experimental studies are needed to address this aspect.

CSF samples were collected from patients treated with an EVD; thus, our CSF findings mainly is related to this selected subgroup of SAH patients. In addition, SAH patients with intraventricular blood (Fisher grade of IV) could conceivably experience variation in the CSF composition depending on positioning and other manipulations affecting cerebrospinal fluid compartments.

All included patients were treated with tranexamic acid (TXA) (an antifibrinolytic) based on the Danish National Guidelines, which might have an impact on complement activity. Many fibrinolytic compounds work as natural C3 and $\mathrm{C} 5$ convertases, thus generating $\mathrm{C} 3 \mathrm{a}$ and $\mathrm{C} 5 \mathrm{a}$ (active anaphylatoxins) [43]. The use of TXA in SAH patients could potentially influence the LCP initiator levels as well as the activity of the complement system.

In our study, we did not control for the presence of intercurrent infection; neither did we measure other markers of inflammation in plasma or CSF. Although $\mathrm{SAH}$ is associated with a marked systemic inflammatory response $[44,45]$, infection, such as pneumonia in mechanically ventilated patients, could also contribute to inflammation and potentially affect the relationship between plasma LCP initiator levels, DCI, and functional outcome. By contrast, the CSF levels of LCP initiators probably represented the true inflammatory response to $\mathrm{SAH}$ in and of itself since ventriculostomy-related infection is rare during the first week after insertion [46].

Additionally, a longitudinal analytical approach results in a high-resolution due to frequent repeated measures, which eases the process of visualizing on which time point (day) potential differences between groups occur. However, this analytical approach limits the likelihood of finding significant differences between groups on a specific day. Thus, in our study $p$ values for individual days are less important than the visualization of the dynamic changes over time.

Finally, this is still a novel investigation field in which our exploratory and retrospective study design was not able to include all potential laboratory and clinical confounders, which may alter LCP profiles in plasma and CSF. Future studies should investigate comorbidities, in particular on-going inflammatory/infectious diseases, neoplastic, paraneoplastic and demyelinating conditions, premorbid hypertension as stated in recent recommendations as well as other potential confounders [42, 47].

\section{Conclusion}

Our study revealed that SAH patients treated with external ventricular drain had elevated levels of LCP initiators in CSF compared with neurologically healthy patients. Intrathecal ficolin-1 and MBL were increased in SAH patients with a poor functional outcome. No association was found between DCI and overall CSF levels of LCP initiators. Future studies should clarify the pathophysiological mechanism behind ficolin-1 in the central nervous system and determine the potential of ficolin- 1 as a prognostic marker of functional outcome. 


\section{Supplementary Information}

The online version contains supplementary material available at https://doi. org/10.1186/s12974-020-01979-y.

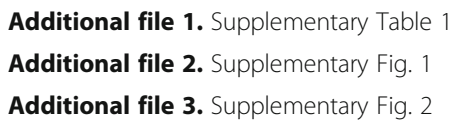

\section{Abbreviations}

BBB: Blood-brain barrier; CNS: Central nervous system; CSF: Cerebrospinal fluid; CT: Computed tomography; DCl: Delayed cerebral ischemia; EBI: Early brain injury; ELISA: Enzyme-linked immunosorbent assay; EVD: External ventricular drain; LCP: Lectin complement pathway; MASP: Mannose-binding lectin associated serine protease; MBL: Mannose-binding lectin; mRS: Modified ranking scale; SAH: Subarachnoid hemorrhage; WFNS: World Federation of Neurosurgical Societies

\section{Acknowledgements}

We are grateful to Mr. Jesper Andresen for laboratory assistance.

\section{Authors' contributions}

JSM and SB had access to all data used in the study and take full responsibility for the integrity of the data. JSM, JLF, and SB take responsibility for the accuracy of the statistical analysis. JSM analyzed data, drafted the manuscript, and revised it critically for intellectual content. CLK collected data and revised the manuscript critically for intellectual content. JLF analyzed data and revised the manuscript critically for intellectual content. PG conceived the study, analyzed laboratory data and revised the manuscript critically for intellectual content, handled funding and supervision. KM conceived and designed the study, revised the manuscript critically for intellectual content, handled funding and supervision. SB conceived and designed the study, collected data, analyzed data, revised the manuscript critically for intellectual content, handled funding and supervision. The authors read and approved the final manuscript.

\section{Funding}

J.S.M. was supported by a 6-month research scholarship by the University Hospital of Copenhagen/Rigshospitalet. C.L.K. received funding from the Ehrenreich Foundation. P.G. was supported by The Danish Research Foundation of Independent Research [DFF-6110-00489], The Svend Andersen Research Foundation and the Novo Nordisk Research Foundation. S.B. received grants from the Research Board at Rigshospitalet, Copenhagen, Denmark and Lundbeckfonden (R212-2015-1987). J.L.F and K.M. report no disclosures.

\section{Availability of data and materials}

The datasets generated and analyzed during the current study are available from the authors on reasonable request and provided a Data Sharing Agreement approved by the Danish Data Protection Agency.

\section{Ethics approval and consent to participate}

Protocols were approved by the Danish Scientific Ethics Committee of the Capital Region (H-3-2013-009 and H-3-2014-073) including amendments for the present study (H-3-2013-009 and H-6-2014-073), and the Danish Data Protection Agency (I-suite \# 02116 and 03279), and registered at clinicaltrials. gov (NCT01791257 and NCT02320539). Informed consent was obtained from the next of kin, the general practitioner and when possible, from the patient.

\section{Consent for publication}

Not applicable.

\section{Competing interests}

The authors declare that they have no competing interest and no conflict of interest with respect to the research, authorship, and publication.

\section{Author details}

'Department of Neuroanaesthesiology, The Neuroscience Centre, Rigshospitalet, University of Copenhagen, Blegdamsvej 3, 2100 Copenhagen $\varnothing$, Denmark. ${ }^{2}$ Section of Biostatistics, Department of Public Health, Faculty of Health and Medical Sciences, University of Copenhagen, Copenhagen,
Denmark. ${ }^{3}$ Laboratory of Molecular Medicine, Department of Clinical Immunology, Section 7631, Rigshospitalet, University of Copenhagen Copenhagen, Denmark. ${ }^{4}$ Department of Clinical Medicine, Faculty of Health and Medical Sciences, University of Copenhagen, Copenhagen, Denmark.

Received: 11 August 2020 Accepted: 5 October 2020

Published online: 12 November 2020

\section{References}

1. Ingall T, Asplund K, Mahonen M, Bonita R. A multinational comparison of subarachnoid hemorrhage epidemiology in the WHO MONICA stroke study. Stroke. 2000;31:1054-61.

2. Johnston SC, Selvin S, Gress DR. The burden, trends, and demographics of mortality from subarachnoid hemorrhage. Neurology. 1998;50:1413-8.

3. de Rooij NK, Linn FH, van der Plas JA, Algra A, Rinkel GJ. Incidence of subarachnoid haemorrhage: a systematic review with emphasis on region, age, gender and time trends. J Neurol Neurosurg Psychiatry. 2007;78:1365-72.

4. Rowland MJ, Hadjipavlou G, Kelly M, Westbrook J, Pattinson KT. Delayed cerebral ischaemia after subarachnoid haemorrhage: looking beyond vasospasm. Br J Anaesth. 2012;109:315-29.

5. Vergouwen MD, Vermeulen M, van Gijn J, et al. Definition of delayed cerebral ischemia after aneurysmal subarachnoid hemorrhage as an outcome event in clinical trials and observational studies: proposal of a multidisciplinary research group. Stroke. 2010;41:2391-5.

6. Adams HP Jr. Early management of the patient with recent aneurysmal subarachnoid hemorrhage. Stroke. 1986;17:1068-70.

7. Foreman B. The Pathophysiology of Delayed Cerebral Ischemia. J Clin Neurophysiol. 2016;33:174-82.

8. Geraghty JR, Testai FD. Delayed cerebral ischemia after subarachnoid hemorrhage: beyond vasospasm and towards a multifactorial pathophysiology. Current atherosclerosis reports. 2017;19:50.

9. Bajic G, Degn SE, Thiel S, Andersen GR. Complement activation, regulation, and molecular basis for complement-related diseases. Embo j. 2015;34:2735-57.

10. Degn SE, Thiel S. Humoral pattern recognition and the complement system. Scand J Immunol. 2013;78:181-93.

11. Garred P, Genster N, Pilely K, et al. A journey through the lectin pathway of complement-MBL and beyond. Immunol Rev. 2016;274:74-97.

12. Degn SE, Hansen AG, Steffensen R, Jacobsen C, Jensenius JC, Thiel S. MAp44, a human protein associated with pattern recognition molecules of the complement system and regulating the lectin pathway of complement activation. J Immunol. 2009;183:7371-8.

13. Fust $G$, Munthe-Fog $L$, Illes $Z$, et al. Low ficolin-3 levels in early follow-up serum samples are associated with the severity and unfavorable outcome of acute ischemic stroke. J Neuroinflammation. 2011;8:185.

14. Zangari $R$, Zanier $E R$, Torgano G, et al. Early ficolin-1 is a sensitive prognostic marker for functional outcome in ischemic stroke. J Neuroinflammation. 2016;13:16.

15. Wang $Z Y$, Sun $Z R$, Zhang $L M$. The relationship between serum mannosebinding lectin levels and acute ischemic stroke risk. Neurochem Res. 2014; 39:248-53.

16. Zhang ZG, Wang C, Wang J, et al. Prognostic value of mannose-binding lectin: 90-day outcome in patients with acute ischemic stroke. Mol Neurobiol. 2015;51:230-9.

17. Llull L, Thiel S, Amaro S, Cervera A, Planas AM, Chamorro A. Ficolin-1 levels in patients developing vasospasm and cerebral ischemia after spontaneous subarachnoid hemorrhage. Mol Neurobiol. 2017;54:6572-80.

18. Sandgaard E, Troldborg A, Lauridsen SV, Gyldenholm T, Thiel S, Hvas AM. Changes in the lectin pathway following intracerebral or spontaneous subarachnoid hemorrhage. Mol Neurobiol. 2019;56:78-87.

19. Zanier ER, Zangari R, Munthe-Fog L, et al. Ficolin-3-mediated lectin complement pathway activation in patients with subarachnoid hemorrhage. Neurology. 2014;82:126-34

20. Cai JY, Sun J, Yu ZQ. Serum mannose-binding lectin levels after aneurysmal subarachnoid hemorrhage. Acta Neurol Scand. 2016;134:360-7.

21. Bache $S$, Rasmussen $R$, Rossing M, Laigaard FP, Nielsen FC, Moller K. MicroRNA Changes in Cerebrospinal Fluid After Subarachnoid Hemorrhage. Stroke. 2017:48:2391-8.

22. Teasdale GM, Drake CG, Hunt W, et al. A universal subarachnoid hemorrhage scale: report of a committee of the World Federation of Neurosurgical Societies. J Neurol Neurosurg Psychiatry. 1988;51:1457. 
23. Fisher CM, Kistler JP, Davis JM. Relation of cerebral vasospasm to subarachnoid hemorrhage visualized by computerized tomographic scanning. Neurosurgery. 1980;6:1-9.

24. van Swieten JC, Koudstaal PJ, Visser MC, Schouten HJ, van Gijn J. Interobserver agreement for the assessment of handicap in stroke patients. Stroke. 1988;19:604-7.

25. Munthe-Fog L, Hummelshoj T, Honore C, et al. Variation in FCN1 affects biosynthesis of ficolin-1 and is associated with outcome of systemic inflammation. Genes Immun. 2012;13:515-22.

26. Munthe-Fog L, Hummelshoj T, Hansen BE, et al. The impact of FCN2 polymorphisms and haplotypes on the Ficolin-2 serum levels. Scand J Immunol. 2007;65:383-92.

27. Munthe-Fog L, Hummelshoj T, Ma YJ, et al. Characterization of a polymorphism in the coding sequence of FCN3 resulting in a Ficolin-3 (Hakata antigen) deficiency state. Mol Immunol. 2008;45:2660-6.

28. Garred P, Madsen HO, Kurtzhals JA, et al. Diallelic polymorphism may explain variations of the blood concentration of mannan-binding protein in Eskimos, but not in black Africans. Eur J Immunogenet. 1992;19:403-12.

29. Jacob A, Alexander JJ. Complement and blood-brain barrier integrity. Mol Immunol. 2014;61:149-52.

30. Brouwer MC, Baas F, van der Ende A, van de Beek D. Genetic variation and cerebrospinal fluid levels of mannose binding lectin in pneumococcal meningitis patients. PLoS One. 2013;8:e65151.

31. Shen $L$, Zheng J, Wang $Y$, et al. Increased activity of the complement system in cerebrospinal fluid of the patients with Non-HIV Cryptococcal meningitis. BMC Infect Dis. 2017;17:7.

32. Kjaeldgaard AL, Pilely K, Olsen KS, et al. Amyotrophic lateral sclerosis: The complement and inflammatory hypothesis. Mol Immunol. 2018;102:14-25.

33. Alexander JJ. Blood-brain barrier (BBB) and the complement landscape. Mol Immunol. 2018;102:26-31.

34. Runza VL, Schwaeble W, Mannel DN. Ficolins: novel pattern recognition molecules of the innate immune response. Immunobiology. 2008;213:297-306.

35. Honore C, Rorvig S, Munthe-Fog L, et al. The innate pattern recognition molecule Ficolin-1 is secreted by monocytes/macrophages and is circulating in human plasma. Mol Immunol. 2008;45:2782-9.

36. De Blasio D, Fumagalli S, Orsini F, et al. Human brain trauma severity is associated with lectin complement pathway activation. J Cereb Blood Flow Metab. 2019;39:794-807.

37. Pedersen ED, Loberg EM, Vege E, Daha MR, Maehlen J, Mollnes TE. In situ deposition of complement in human acute brain ischaemia. Scand J Immunol. 2009;69:555-62.

38. Huang J, Kim LJ, Mealey R, et al. Neuronal protection in stroke by an sLexglycosylated complement inhibitory protein. Science. 1999;285:595-9.

39. Mocco J, Mack WJ, Ducruet AF, et al. Complement component C3 mediates inflammatory injury following focal cerebral ischemia. Circ Res. 2006;99:209-17.

40. Imm MD, Feldhoff PW, Feldhoff RC, Lassiter HA. The administration of complement component C9 augments post-ischemic cerebral infarction volume in neonatal rats. Neurosci Lett. 2002;325:175-8.

41. Orsini F, Fumagalli S, Csaszar E, et al. Mannose-binding lectin drives platelet inflammatory phenotype and vascular damage after cerebral ischemia in mice via IL (Interleukin)-1alpha. Arterioscler Thromb Vasc Biol. 2018;38:2678-90.

42. Chou SH, Macdonald RL, Keller E, Unruptured Intracranial A, Investigators SCP. Biospecimens and molecular and cellular biomarkers in aneurysmal subarachnoid hemorrhage studies: common data elements and standard reporting recommendations. Neurocrit Care. 2019;30:46-59.

43. Amara U, Flierl MA, Rittirsch D, et al. Molecular intercommunication between the complement and coagulation systems. J Immunol. 2010;185:5628-36.

44. Krzyzewski RM, Klis KM, Kwinta BM, Stachura K, Guzik TJ, Gasowski J. High Leukocyte Count and Risk of Poor Outcome After Subarachnoid Hemorrhage: A Meta-Analysis. World Neurosurg. 2020;135:e541-7.

45. Schuss P, Hadjiathanasiou A, Brandecker S, Guresir A, Vatter H, Guresir E. Elevated C-reactive protein and white blood cell count at admission predict functional outcome after non-aneurysmal subarachnoid hemorrhage. J Neurol. 2018;265:2944-8.

46. Mounier R, Birnbaum R, Cook F, et al. Natural history of ventriculostomyrelated infection under appropriate treatment and risk factors of poor outcome: a retrospective study. J Neurosurg. 2018:1-10.

47. Jaja BNR, Saposnik G. Lingsma HF, et al. Development and validation of outcome prediction models for aneurysmal subarachnoid haemorrhage: the SAHIT multinational cohort study. 2018;360:j5745.

\section{Publisher's Note}

Springer Nature remains neutral with regard to jurisdictional claims in published maps and institutional affiliations.
Ready to submit your research? Choose BMC and benefit from:

- fast, convenient online submission

- thorough peer review by experienced researchers in your field

- rapid publication on acceptance

- support for research data, including large and complex data types

- gold Open Access which fosters wider collaboration and increased citations

- maximum visibility for your research: over $100 \mathrm{M}$ website views per year

At BMC, research is always in progress.

Learn more biomedcentral.com/submissions 